\title{
Calculating the force-dependent unbinding rate of biological macromolecular bonds from force-ramp optical trapping assays
}

\section{Apurba Paul}

Clemson University

Joshua Alper ( $\square$ alper@clemson.edu )

Clemson University

\section{Research Article}

Keywords: force, dissociation, data, bonds, rate, protein

Posted Date: September 29th, 2021

DOl: https://doi.org/10.21203/rs.3.rs-933021/v1

License: (c) (1) This work is licensed under a Creative Commons Attribution 4.0 International License.

Read Full License

Version of Record: A version of this preprint was published at Scientific Reports on January 7th, 2022. See the published version at https://doi.org/10.1038/s41598-021-03690-1. 


\section{Calculating the force-dependent unbinding rate of}

\section{2 biological macromolecular bonds from force-ramp}

\section{optical trapping assays}

11 Abstract

12 The non-covalent biological bonds that constitute protein-protein or protein-ligand interactions

13 play crucial roles in many cellular functions, including mitosis, motility, and cell-cell adhesion.

14 The effect of external force $(F)$ on the unbinding rate $\left(k_{\mathrm{off}}(F)\right)$ of macromolecular interactions

15 is a crucial parameter to understanding the mechanisms behind these functions. Optical

16 tweezer-based single-molecule force spectroscopy is frequently used to obtain quantitative

17 force-dependent dissociation data on slip, catch, and ideal bonds. However, analyses of this 
18 data using dissociation time or dissociation force histograms often quantitatively compare

19 bonds without fully characterizing their underlying biophysical properties. Additionally, the results of histogram-based analyses can depend on the rate at which force was applied during the experiment and the experiment's sensitivity. Here, we present an analytically derived cumulative distribution function-like approach to analyzing force-dependent dissociation force spectroscopy data. We demonstrate the benefits and limitations of the technique using stochastic simulations of various bond types. We show that it can be used to obtain the detachment rate and force sensitivity of biological macromolecular bonds from force spectroscopy experiments by explicitly accounting for loading rate and noisy data. We also discuss the implications of our results on using optical tweezers to collect force-dependent dissociation data.

The weak non-covalent bonds that constitute protein-protein and protein-ligand interactions underlie nearly all cellular functions ${ }^{1}$. For example, the physical chemistry of protein-protein interactions is fundamental to the molecular mechanisms of the cytoskeleton ${ }^{2}$. Kinesin and dynein motor proteins precisely regulate and coordinate changes in motor-filament binding affinity as a function of their mechanochemical cycles as they walk along microtubules, and quantitative models of motors must explicitly account for the effects of external forces $(F)$ on the filament unbinding rate constant $\left(k_{\mathrm{off}}(F)\right)^{2}$. Beyond motors, examples of force-dependent unbinding rate constants are ubiquitous in biological systems, including cell-cell adhesion ${ }^{3-5}$, mechanotransduction $^{6,7}$, DNA polymerases and helicases ${ }^{8,9}$, membrane-surface adhesion $^{10}$, selectin-ligand ${ }^{11}$, and antibody-antigen complexes $^{12-14}$.

40 Weak non-covalent bonds between biological macromolecules can be classified into three 41 principal categories ${ }^{15}$. Slip bonds have a lifetime that decreases with the increasing applied 
force $^{16}$. Catch bonds have a lifetime that increases with applied force ${ }^{10,17}$. Ideal bonds have a

43 lifetime that is independent of applied force ${ }^{10}$. Many biomolecular interactions have been

44 characterized using single-molecule techniques and understood in the context of these models.

45 Single-molecule force spectroscopy experiments involving the force-dependent unbinding of motor proteins ${ }^{18}$, microtubule- and actin-associated proteins ${ }^{19-21}$, focal adhesion proteins ${ }^{22,23}$, and many others ${ }^{24-26}$, are commonly done using optical tweezers to apply a constant (i.e., a step function in time) or linearly increasing force (e.g., a force ramp starting a zero upon binding and increasing until detachment) to the molecules (Figure 1a). Data calculated from

50 force spectroscopy traces frequently include the bound time and unbinding force (Figure 1b).

51 For example, recent single-molecule experiments on cytoplasmic dynein motor proteins show

52 both catch bond and slip-ideal bond behaviors ${ }^{27,28}$ and that kinesin exhibits force-dependent stepping velocities and microtubule unbinding rates that are a function of force direction ${ }^{29}$.

54 Multiple techniques exist to analyze this data ${ }^{30}$, including a recent work extending classical analysis techniques to characterize motor proteins pulling cargo from a stationary optical trap ${ }^{31}$.
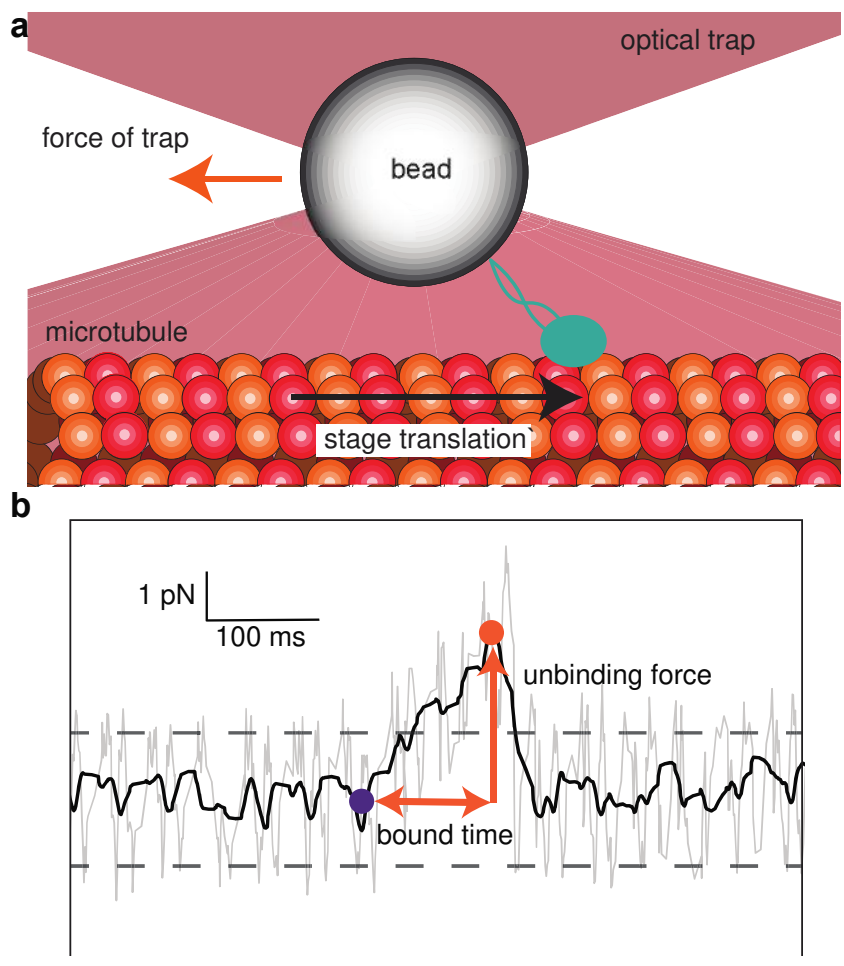

optical trapping assay.

This report presents a cumulative distribution function approach to these analysis techniques that unambiguously characterizes biological macromolecular slip, ideal, and catch-bond forcedependent unbinding. It deconvolutes the effects of how the experiment was performed with the unbinding force and time probably distributions, and it accounts for noise-based detection limits. We demonstrate the method's ability to extract the molecular parameters from forceramp optical trapping assay data, including the zero-force unbinding rate constant, forcedependent rate constants, and force sensitivities, using simulated data from various bond types.

Theory

Consider a non-covalent, biological macromolecular bond, like one that underlies a proteinprotein interaction. Based on classical thermodynamic models, the system has an unbinding constant, $k_{\text {off }}$, at quasi-thermodynamic equilibrium (for bonds subject to loading that is slow compared to the timescales of force equilibrium and thermal fluctuations). In the absence of force and in a dilute solution, the average lifetime of a protein-protein interaction bond is the inverse of its unbinding constant, $\left\langle\tau_{\text {off }}\right\rangle=1 / k_{\text {off }}$. We consider an ensemble with $N$ independent bonds formed at $t=0$, of which $n(t)$ of them remain bound at time $t$. The unbinding kinetics of the ensemble, assuming bonds do not reform after breaking, are

$$
\frac{d n}{d t}=-k_{\mathrm{off}}(F(t)) n
$$

In general, the unbinding constant $k_{\text {off }}(F(t))$ is a function of the force applied to the bond, and we consider the case that each bond is subject to a time-varying external force, $F(t)$, e.g., as exerted by an optical tweezer. 
By separating the variables and integrating equation (1), we find a cumulative distributionlike function for $n(t)$,

$$
n(t)=N e^{-\int k_{\mathrm{off}}(F(t)) \mathrm{d} t}
$$

82 The functional form of $k_{\text {off }}(F(t))$ depends on the bond type and how force changes with time, e.g., a linearly increasing force $\operatorname{ramp}(F(t)=f t)$ where $f$, the time rate of change of applied force, is constant. By applying models that relate the unbinding constant and force, we can complete the integration and obtain a functional form for the number of unbroken bonds as a function of time, $n(t)$.

Slip bonds

The unbinding constant of a slip bond, $k_{\mathrm{off}, \mathrm{s}}$, increases exponentially with force per Bell's $\operatorname{model}^{16}$

$$
k_{\mathrm{off}, \mathrm{s}}(F(t))=k_{0, \mathrm{~s}} e^{\frac{F(t)}{F_{\mathrm{s}}}}
$$

where $k_{0, \mathrm{~s}}$ is the force-free unbinding constant and $F_{\mathrm{S}}$ is the force sensitivity of the slip bond.

Slip bonds with small $F_{\mathrm{s}}$ as compared to the applied load are more force-sensitive than those with large $F_{\mathrm{s}}$. Thus, for a linearly increasing force ramp,

$$
k_{\mathrm{off}, \mathrm{s}}(t)=k_{0, s} e^{\frac{f t}{F_{\mathrm{s}}}}
$$

Therefore, equation (2) is

$$
n(t)=N e^{-\int k_{0, s} e^{\frac{f t}{F_{\mathrm{s}}}} \mathrm{d} t}=N e^{-\frac{F_{\mathrm{s}} k_{0, s}}{f}\left(e^{\frac{f t}{F_{\mathrm{s}}}}-1\right)},
$$

97 for a slip bond.

98 However, the fastest unbinding events may not be observable above experimental noise or 99 may not be within the time sensitivity of force spectroscopy data. For example, 100 binding/unbinding events in a linear force ramp experiment (Figure 1b) are only observable if 101 the force at the time of dissociation is significantly larger than the amplitude of the thermal 
102 fluctuations of a bead in the trap times the effective stiffness of the trap, $F_{\text {noise }}$ Therefore, we

103 introduce a time scale, $t_{0}$, representing the shortest observable event in a linear force ramp

104 experiment, $t_{0}=F_{\text {noise }} / f$. Thus, the total number of observable binding events is

$$
n_{0}=N e^{-\frac{F_{s} k_{0, s}}{f}\left(e^{\frac{f t_{0}}{F_{\mathrm{s}}}}-1\right)} .
$$

106 Due to the experimental noise, this analysis suggests that

$$
N-n_{0}=n_{0} e^{\frac{F_{s} k_{0, \mathrm{~s}}}{f}\left(e^{\frac{f t_{0}}{F_{\mathrm{s}}}}-1\right)}-n_{0}=n_{0}\left(e^{\frac{F_{\mathrm{s}} k_{0, \mathrm{~s}}}{f}\left(e^{\frac{f t_{0}}{F_{\mathrm{s}}}}-1\right)}-1\right)
$$

unbinding events occur in a time less than $t_{0}$ and are missing from the observable dataset. Thus, to characterize the physical properties of a slip bond, one can record the number of bonds that

110 remain unbroken as a function of time, and fit the data to

$$
n(t)=n_{0} e^{-\frac{F_{s} k_{0, s}}{f}\left(\mathrm{e}^{\frac{f t}{F_{s}}}-\mathrm{e}^{\frac{f t_{0}}{F_{\mathrm{s}}}}\right)},
$$

112 where the slip bond characteristics, $F_{\mathrm{S}}$ and $k_{0, s}$, are the fitting parameters. $n(t)$, and therefore

$113 F_{\mathrm{s}}$ and $k_{0, \mathrm{~s}}$, strongly depend on the loading rate, $f$, of the experiment. While $f=\kappa v$ can be

114 controlled by changing with the stage translation rate, $v$, in a fixed-beam optical tweezer assay

115 (Figure 1a) or trap translation in a fixed-stage optical tweezer assay, and the trap stiffness, $\kappa_{\text {trap }}$,

116 the loading rate is additionally complicated by the finite stiffness of the system, including

117 linking molecules, $\kappa_{\text {system }}$, where $\frac{1}{\kappa}=\frac{1}{\kappa_{\text {trap }}}+\frac{1}{\kappa_{\text {system }}}$.

118 Ideal bonds

119 The unbinding constant of an ideal bond, $k_{\text {off,i }}$, does not change with force. Hence, even when

120 the applied force changes with time, the unbinding constant remains constant. In that case, the unbinding constant is $k_{\mathrm{off}, \mathrm{i}}(t)=k_{0, \mathrm{i}}$ and equation (2) becomes

$$
n(t)=N e^{-k_{0, \mathrm{i}} t}
$$




$$
n(t)=n_{0} e^{-k_{0, \mathrm{i}}\left(t-t_{0}\right)},
$$

125 where $n_{0}$ is the total number of observable binding events. $n(t)$ is not a function of the loading

126 rate, $f$, in the ideal bond case, and ideal bonds are special cases of slip bonds where the bond

127 is entirely insensitive to force, i.e., for the limit when $F_{\mathrm{S}} \rightarrow \infty$ (Supplementary Information).

128 Slip-ideal bonds

129 Recent data suggests that certain protein-protein interactions may be best modeled as a slip-

130 ideal bond ${ }^{28}$. The force-dependent unbinding constant of a slip-ideal bond, $k_{\text {off,s-i }}(F(t))$,

131 increases exponentially with force per Bell's model ${ }^{16}$ up to the slip-ideal transition force, $F_{\mathrm{s}-\mathrm{i}}$,

132 beyond which, it behaves as an ideal bond (Figure 2a). Therefore, the simplest mathematical model for the force-dependent unbinding constant of a slip-ideal bond is a piecewise function,

$$
k_{\mathrm{off}, \mathrm{s}-\mathrm{i}}(F(t))= \begin{cases}k_{0, \mathrm{~s}} e^{\frac{F(t)}{F_{\mathrm{s}}}} & F<F_{\mathrm{s}-\mathrm{i}} . \\ k_{0, \mathrm{i}} & F \geq F_{\mathrm{s}-\mathrm{i}}\end{cases}
$$

135 While there appear to be four independent parameters: the ideal bond's unbinding constant, the

136 slip bond's unbinding constant and force sensitivity, and the slip-ideal transition force, $F_{\mathrm{s}-\mathrm{i}}$,

137 they are related by $k_{0, \mathrm{i}}=k_{0, \mathrm{~s}} e^{\frac{F_{\mathrm{s}-\mathrm{i}}}{F_{\mathrm{s}}}}$, or equivalently $F_{\mathrm{s}-\mathrm{i}}=\alpha F_{\mathrm{s}}$ where $\alpha=\ln \frac{k_{0, \mathrm{i}}}{k_{0, \mathrm{~s}}}$, due to the 138 continuity condition at $F=F_{\mathrm{s}-\mathrm{i}}$. Thus, the slip-ideal model can be written as either

$$
k_{\mathrm{off}, \mathrm{S}-\mathrm{i}}(F(t))= \begin{cases}k_{0, s} e^{\frac{F(t)}{F_{s}}} & F<F_{s-i} \\ k_{0, s} e^{\frac{F_{s-i}}{F_{S}}} & F \geq F_{s-i}\end{cases}
$$

140 or

$$
k_{\mathrm{off}, \mathrm{s}-\mathrm{i}}(F(t))= \begin{cases}k_{0, s} e^{\frac{F(t)}{F_{s}}} & F<\alpha F_{s} . \\ k_{0, s} e^{\alpha} & F \geq \alpha F_{S}\end{cases}
$$



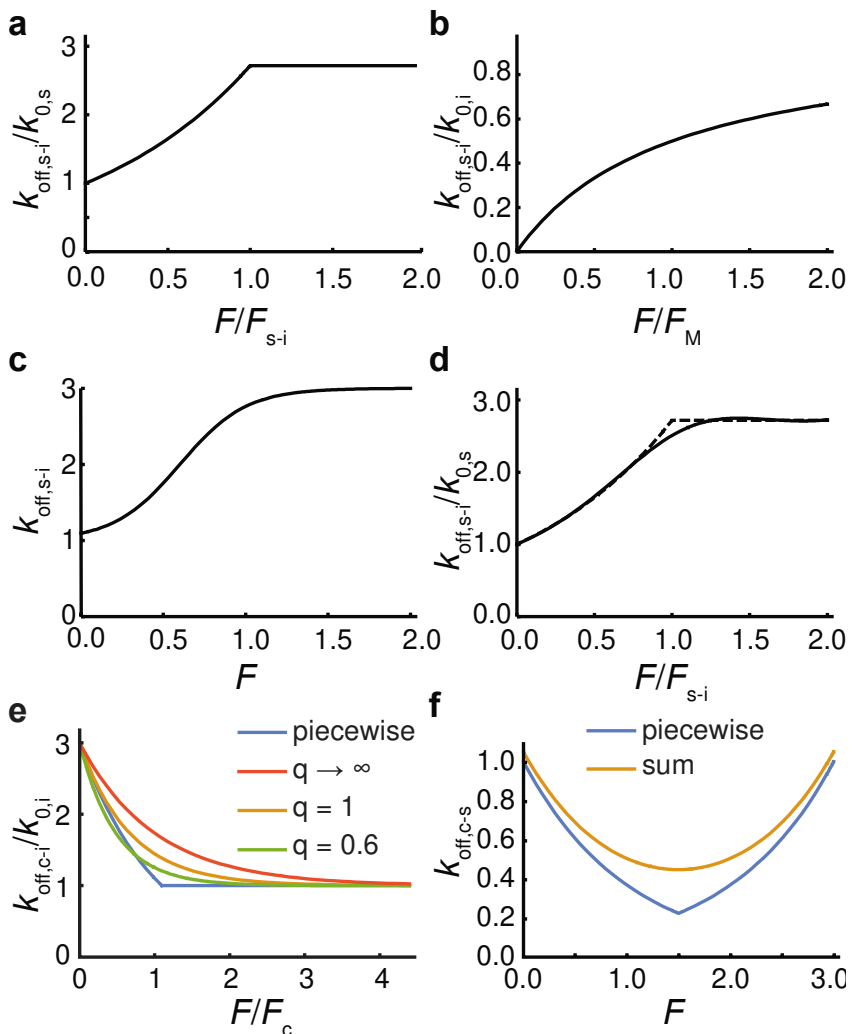

143 Figure 2. Analytical models for the force-dependent unbinding constant of slip-ideal, catch-ideal, and catch-slip bonds.

145 Unfortunately, the piecewise nature of equation (10) does not lend itself well to further 146 mathematical analysis. However, there are multiple approaches to approximating slip-ideal

147 bonds, which enable further analysis and ultimately fitting data to equation (2). These 148 approaches include a Michaelis-Menten or Langmuir absorption-like function (Figure 2b) ${ }^{31}$, a 149 sigmoidal model approximation (Figure 2c), and a rational interpolation ${ }^{32,33}$ approach (Figure

150 2c) that uses the RationalInterpolation function in Mathematica ${ }^{34}$, for example. See the

151 Supporting Information for a more detailed discussion on these approximations of slip-ideal 152 bonds. 
154 The unbinding constant of the catch bond, $k_{\text {off,c }}$, decreases exponentially with force per Bell's 155 model $^{16}$,

$$
k_{\mathrm{off}, \mathrm{c}}(F(t))=k_{0, \mathrm{c}} e^{-\frac{F(t)}{F_{\mathrm{c}}}}
$$

157 So, for a constant loading and $n_{0}$ observable events, equation (2) becomes

$$
n(t)=N e^{-\frac{F_{\mathrm{c}} k_{0, \mathrm{c}}}{f}\left(1-\mathrm{e}^{-\frac{f t}{F_{\mathrm{c}}}}\right)}
$$

or

161 However, this is a relatively limited model because it models the unbinding rate constant as going to zero as $F$ goes to infinity. Equation (11) suggests that a catch bond will never break under an arbitrarily high load, which is non-physical. Therefore, equation (11) is only valid for low force. All known catch-bonds invariably transform into slip or ideal bonds as the forces increase beyond a critical value ${ }^{35,36}$. To capture the high-loading case, one can model all catch

166 bonds as catch-slip or catch-ideal bonds.

\section{Catch-ideal bonds}

168 A simple, theoretically appealing way to correct for the physical limitation of the catch bond 169 is with a catch-ideal bond. A catch-ideal bond can be modeled as a bond that behaves like a 170 catch bond under low loading and an ideal bond after the force crosses the catch-ideal transition

171 force, $F_{\mathrm{c}-\mathrm{i}}$. The unbinding constant of the catch-ideal bond, $k_{\mathrm{off}, \mathrm{c}-\mathrm{i}}$ (Figure 2e), is

$$
k_{\mathrm{off}, \mathrm{c}-\mathrm{i}}(F(t))= \begin{cases}k_{0, \mathrm{c}} e^{-\frac{F(t)}{F_{\mathrm{c}}}} & F<F_{\mathrm{c}-\mathrm{i}} \\ k_{0, \mathrm{c}} e^{-\frac{F_{\mathrm{c}-\mathrm{i}}}{F_{\mathrm{c}}}} & F \geq F_{\mathrm{c}-\mathrm{i}}\end{cases}
$$

or 


$$
k_{\mathrm{off}, \mathrm{c}-\mathrm{i}}(F(t))= \begin{cases}k_{0, \mathrm{c}} e^{-\frac{F(t)}{F_{\mathrm{c}}}} & F<F_{\mathrm{c}-\mathrm{i}}, \\ k_{0, \mathrm{i}} & F \geq F_{\mathrm{c}-\mathrm{i}}\end{cases}
$$

175 where only three of the four parameters are independent: $k_{0, \mathrm{i}}=k_{0, \mathrm{c}} e^{-\frac{F_{\mathrm{c}-\mathrm{i}}}{F_{\mathrm{c}}}}$ or $F_{\mathrm{c}-\mathrm{i}}=F_{\mathrm{c}} \ln \frac{k_{0, \mathrm{c}}}{k_{0, \mathrm{i}}}$, due 176 to the continuity condition at $F=F_{\mathrm{c}-\mathrm{i}}$.

177 We can approximate equation (13) with a sigmoidal function,

$$
k_{\mathrm{off}, \mathrm{c}-\mathrm{i}}(F(t))=\left(k_{0, \mathrm{c}}-k_{0, \mathrm{i}}\right) e^{-\frac{F(t)}{\left(1-\left(\frac{k_{0, \mathrm{i}}}{k_{0, \mathrm{c}}}\right)^{q}\right) F_{\mathrm{c}}}}+k_{0, \mathrm{i}} .
$$

179 At zero force, the sigmoidal approximation behaves like a catch bond, $k_{\text {off,c-i }}(0)=k_{0, \mathrm{c}}$, and $k_{\mathrm{off}, \mathrm{c}-\mathrm{i}}(F \rightarrow \infty) \rightarrow k_{0, \mathrm{i}}$ as force goes to infinity. Equation (14) shares the same initial slope,

$181 \frac{\partial k_{\text {off,c-i }}}{\partial F}(0)=-\frac{k_{0, \mathrm{c}}}{F_{\mathrm{c}}}$, with equation (13) if $q=1$, and it represents a simpler approximation, that $182 k_{\mathrm{off}, \mathrm{c}-\mathrm{i}}(F(t))=\left(k_{0, \mathrm{c}}-k_{0, \mathrm{i}}\right) e^{-\frac{F(t)}{F_{\mathrm{c}}}}+k_{0, \mathrm{i}}$, for q goes to infinity (Figure 2e). The best fit of equation (14) to the piecewise function (equation (13), found with a least-squares regression, becomes

186

$$
\left.n(t)=\mathrm{N} \mathrm{e}^{\left(\frac{\left(1-\left(\frac{k_{0, \mathrm{i}}}{k_{0, \mathrm{c}}}\right)^{q}\right) F_{\mathrm{c}}\left(k_{0, \mathrm{c}}-k_{0, \mathrm{i}} \mathrm{i}\right.}{f}\right.}\left(1-\mathrm{e}^{-\frac{f t}{\left(1-\left(\frac{k_{0, \mathrm{i}}}{k_{0, \mathrm{c}}}\right)^{q}\right) F_{\mathrm{c}}}}\right)-\mathrm{k}_{0, \mathrm{i}} t\right)
$$

187 or

188

$$
n(t)=n_{0} e^{\left(\frac{\left(1-\left(\frac{k_{0, \mathrm{i}}}{k_{0, \mathrm{c}}}\right)^{q}\right) F_{\mathrm{c}}\left(k_{0, \mathrm{c}}-k_{0, \mathrm{i}}\right)}{f}\left(\mathrm{e}^{-\frac{f t_{0}}{\left(1-\left(\frac{k_{0, \mathrm{i}}}{k_{0, \mathrm{c}}}\right)^{q}\right)_{F_{\mathrm{c}}}}-\mathrm{e}}-\frac{f t}{\left(1-\left(\frac{k_{0, \mathrm{i}}}{k_{0, \mathrm{c}}}\right)^{q}\right)}\right)-k_{0, i}\left(t-t_{0}\right)\right.} .
$$

\section{Catch-slip bonds}

190 While the catch-ideal bond is appealing, there is a stronger theoretical basis of ${ }^{35,37}$ and

191 experimental evidence for $^{38}$ catch-slip bonds. The conceptual model of a catch-slip bond 
192 suggests a transition from catch bond to slip bond behavior when the force exceeds a critical 193 catch-slip transition value, $F_{c-s}$. A five-parameter piecewise function can mathematically model 194 catch-slip bonds (Figure 2f),

$$
k_{\mathrm{off}, \mathrm{c}-\mathrm{s}}(F(t))= \begin{cases}k_{0, \mathrm{c}} e^{-\frac{F}{F_{\mathrm{c}}}} & F \leq F_{\mathrm{c}-\mathrm{s}} \\ k_{0, \mathrm{~s}} e^{\frac{F}{F_{\mathrm{s}}}} & F>F_{\mathrm{c}-\mathrm{s}}\end{cases}
$$

196 where only four parameters are independent because $k_{0, \mathrm{c}} e^{-\frac{F_{\mathrm{c}-\mathrm{s}}}{F_{\mathrm{c}}}}=k_{0, \mathrm{~s}} e^{\frac{F_{\mathrm{c}-\mathrm{s}}}{F_{\mathrm{s}}}}$, or equivalently $197 \quad F_{\mathrm{c}-\mathrm{s}}=\frac{F_{\mathrm{c}} F_{\mathrm{s}}}{F_{\mathrm{c}}+F_{\mathrm{s}}} \ln \left(\frac{k_{0, \mathrm{c}}}{k_{0, \mathrm{~s}}}\right)$, due to the continuity condition at $F=F_{\mathrm{c}-\mathrm{s}}$.

198 Catch-slip bonds have been modeled as dissociating through one of two independent 199 pathways: the catch bond pathway at relatively low force and short times, and the slip bond 200 pathway at relatively high force and long times ${ }^{10}$. The analytical expression for this model is

$$
k_{\mathrm{off}, \mathrm{c}-\mathrm{s}}(F(t))=k_{0, \mathrm{c}} e^{-\frac{F}{F_{\mathrm{c}}}}+k_{0, \mathrm{~s}} e^{\frac{F}{F_{\mathrm{s}}}}
$$

202 provided $\frac{k_{0, \mathrm{c}}}{F_{\mathrm{c}}}<\frac{k_{0, \mathrm{~s}}}{F_{\mathrm{s}}}$ (Figure $\left.2 \mathrm{f}\right)$. In this case, the catch-slip transition force, $F_{\mathrm{c}-\mathrm{s}}=\frac{F_{\mathrm{c}} F_{\mathrm{s}}}{F_{\mathrm{c}}+F_{\mathrm{s}}} \ln \left(\frac{k_{0, \mathrm{c}} F_{\mathrm{s}}}{k_{0, \mathrm{~s}} F_{\mathrm{c}}}\right)$,

203 occurs when the first derivative of equation (17) with respect to force is zero. For constant 204 loading rate, equation (2) becomes

$$
n(t)=N e^{-\left(\frac{F_{\mathrm{s}} k_{0, s}\left(e^{\frac{f t}{F_{\mathrm{S}}}}-1\right)+F_{\mathrm{c}} k_{0, c}\left(1-e^{-\frac{f t}{F_{\mathrm{c}}}}\right)}{f}\right)}
$$

or

$$
n(t)=n_{0} e^{-\left(\frac{F_{\mathrm{s}} k_{0, \mathrm{~s}}\left(e^{\frac{f t}{F_{\mathrm{s}}}}-e^{\frac{f t_{0}}{F_{\mathrm{s}}}}\right)+F_{\mathrm{c}} k_{0, \mathrm{c}}\left(e^{-\frac{f t_{0}}{F_{\mathrm{c}}}}-e^{-\frac{f t}{F_{\mathrm{c}}}}\right)}{f}\right)} .
$$


209 We stochastically simulated the unbinding of non-covalent, biomolecular bonds in

210 MATLAB $^{39}$ using the Gillespie algorithm ${ }^{40}$. We considered a system with $N$ independently

211 attached bonds at time $t=0$ and applied a constantly increasing force (with constant loading

212 rate $f$ ) to each bond. We simulated the time at which each bond, $i=1 \rightarrow N$, broke,

$213 t_{i}=t_{i-1}+\tau_{i}$, where

$$
\tau_{i}=-\frac{\ln (r)}{n\left(t_{i}\right) k_{\mathrm{off}}\left(F\left(t_{i}\right)\right)}
$$

215 per standard implementation of the Gillespie algorithm ${ }^{41}, r$ is a random number between 0 and

2161 pulled from a uniform distribution, $n\left(t_{i}\right)=N-i$ is the number of bonds that remain attached

217 after time $t_{i}$, and $k_{\text {off }}\left(F\left(t_{i}\right)\right)$ is the force-dependent unbinding constant.

\section{Results and discussion}

219 We demonstrate the benefits of fitting single-molecule unbinding data to the cumulative 220 distribution function-like $n(t)$ over more traditional histogram analysis using simulated data.

221 We do so, rather than using example experimental data, to demonstrate the power of this analysis on datasets for which we know the underlying biophysical nature of the bonds because we set them in the simulations. We use slip bonds and catch-slip bonds as examples (for brevity) because they are commonly reported bond types in the literature. However, the analysis could be easily extended to the other bond types and approximations of their functional forms, as discussed above and the Supporting Information.

Slip bonds

228 We simulated $N=1000$ slip bonds with unloaded unbinding rate $k_{0, \mathrm{~s}}=1 \mathrm{~s}^{-1}$ and force sensitivity $F_{S}=1 \mathrm{pN}$ subjected to no loading $\left(k_{\mathrm{off}, \mathrm{S}}(F=0)=k_{0, \mathrm{~s}}\right.$ per equation $(3)$ and $n(t)=$ 
$230 \mathrm{~N} e^{-k_{0, s} t}$, Figure 3a) and with linearly increasing loads $\left(k_{\mathrm{off}, \mathrm{s}}(F=f t)\right.$ per equation (4) and

$231 n(t)$ per equation (5), Figure 3a). By comparing histograms of the time to detach for 1000

232 simulated bonds (Figure 3b), we found that the time to detachment distribution was a strong

233 function of loading rate. With no external load ( $F=0$, Figure $3 \mathrm{~b}$, upper left $)$, the histogram

234 took the form of exponential decay, as expected for a single kinetic process. However, the

235 characteristic form of the histogram became increasing Gaussian-like (though not strictly

236 Gaussian) as the loading rate, $f$, increased (Figure $3 b$ ).

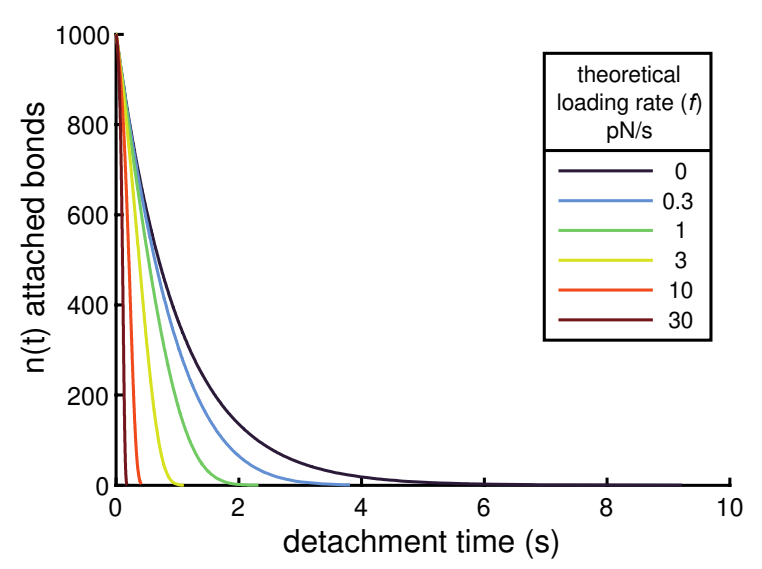

C

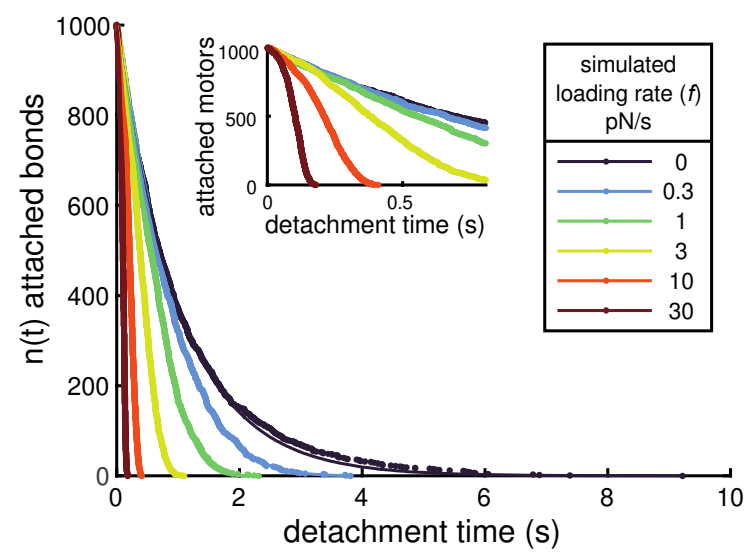

b

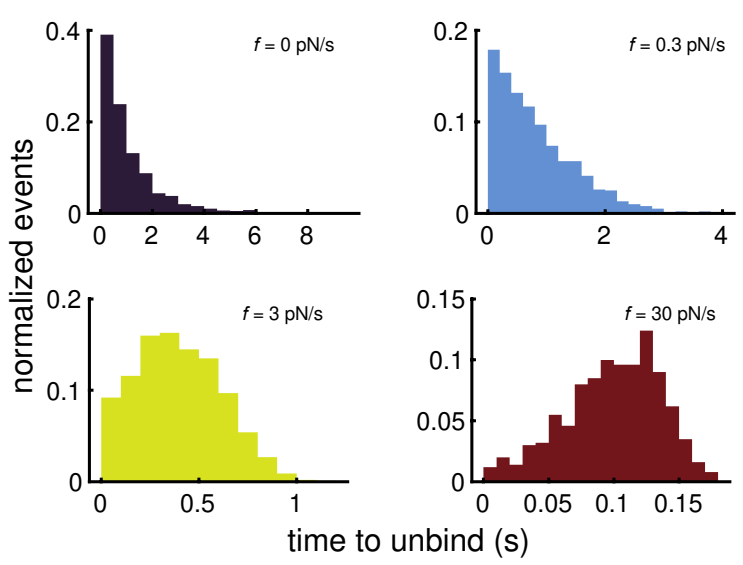

d

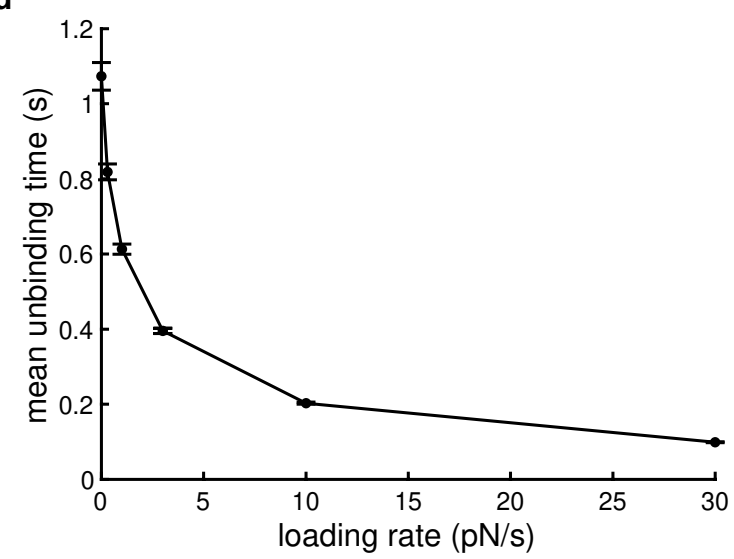

Figure 3. Calculating the force-dependent unbinding rate for slip bonds.

We also plotted the force-free and force-dependent dissociation of simulated slip bonds

240 (same data as Figure $3 b$ ) as a function of their time to dissociate $(n(t))$. We fit these data to

$241 n(t)=\mathrm{N} e^{-k_{0, \mathrm{~s}} t}$ and equation (5) (Figure 3c, Table S2, and Supporting Information for details),

242 respectively. We repeated the simulations 50 times and calculated the mean fitting parameters 
for each loading rate (Table 1). We found that the means of the fit parameters, $k_{0, s}$ and $F_{0}$, were

244 not significantly different from the corresponding parameters used in the simulations (P values

$245>0.05$ in all cases, Table 1, two-tailed t-tests). Thus, our cumulative distribution function-like

246 analysis uniquely and accurately determined the underlying biophysical parameters associated

247 with slip bonds at each loading rate, suggesting one only needs to collect one such data set to

248 characterize a slip bond.

249 Table 1: Mean fit parameters from simulated slip bond dissociation data sets

\begin{tabular}{|c|c|c|c|c|}
\hline $\begin{array}{c}\text { Loading rate } \\
(\mathrm{pN} / \mathrm{s})\end{array}$ & $\begin{array}{c}k_{0, \mathrm{~s}}\left(\mathrm{~s}^{-1}\right)(\mathrm{fit} \pm \\
\text { s.e.m. })\end{array}$ & $\mathrm{P}$ value & $\begin{array}{c}F_{\mathrm{s}}(\mathrm{pN})(\mathrm{fit} \pm \\
\text { s.e.m. })\end{array}$ & $\mathrm{P}$ value \\
\hline $\begin{array}{c}\text { Simulation } \\
\text { parameter }\end{array}$ & 1 & - & 1 & - \\
\hline 0 & $1.009 \pm 0.005$ & 0.06 & - & - \\
\hline 0.3 & $0.990 \pm 0.008$ & 0.21 & $1.019 \pm 0.038$ & 0.62 \\
\hline 1 & $0.995 \pm 0.009$ & 0.58 & $0.998 \pm 0.015$ & 0.88 \\
\hline 3 & $1.018 \pm 0.008$ & 0.06 & $1.010 \pm 0.008$ & 0.25 \\
\hline 10 & $0.992 \pm 0.012$ & 0.48 & $0.997 \pm 0.006$ & 0.67 \\
\hline 30 & $0.996 \pm 0.016$ & 0.81 & $1.002 \pm 0.006$ & 0.69 \\
\hline
\end{tabular}

250

251 We also calculated the mean detachment time for each force-ramp loading rate condition

252 (Figure 3d). In the unloaded case, the mean unbinding time was $1.073 \mathrm{~s}$ for the example data

253 in Figure 3b, which was similar to but not a particularly great predictor of (7.3\% error) the

254 expected value of $\frac{1}{k_{0, \mathrm{~s}}}(1 \mathrm{~s}$, for this simulation) for a first-order kinetic process (Supporting

255 Information). However, when extending this analysis to the force-dependent unbinding

256 properties, we found that the mean of the detachment time decreased with increasing loading

257 rate (Figure 3d). Therefore, the underlying biophysical parameters of the bond are inaccessible

258 to histogram analysis without explicitly accounting for the loading rate, and even then, it 
requires collecting large data sets at multiple loading rates (Supporting Information for more details).

261 The effect of experimental noise on slip bonds

262 In an experiment (e.g., Figure 1a), it is impossible to distinguish single-molecule dissociation 263 data for short times from the noise associated with the experiments. The noise "hides" 264 detachment events if the detected data are smaller than four to five times the standard deviation 265 of the noise. Selecting a detection threshold as high as five times the standard deviation is necessary to avoid misinterpreting the noise as a molecular unbinding event because the data collection rate necessary for high temporal resolution necessitates capturing many data points. For example, one would expect to misinterpret noise as a binding event 380 times with a $4 \sigma$ threshold and 3.4 times with a $5 \sigma$ threshold, on average, for 5 minutes of data collection at a $20 \mathrm{kHz}$ sampling rate $(6,000,000$ data points $)$. Depending on the total number of events within the measurement time, but frequently of order $10^{2}-10^{3}$ events, hundreds of false-positive events could lead to a significant misinterpretation of the results. Given a loading rate, this detection threshold sets and effective minimum time for events, a detection threshold of $t_{0}$, as described 274 above.

275 We applied a detection threshold of $t_{0}=50 \mathrm{~ms}$ to the example simulated data in Figure $3 \mathrm{~b}$, and we found that the mean unbinding time in the unloaded case increased to $1.130 \mathrm{~s}$ from $1.073 \mathrm{~s}$ with $t_{0}=0 \mathrm{~ms}(\mathrm{P}$ value $=0.28$, two-tailed t-test $)$. However, in the case of $f=30 \mathrm{pN} / \mathrm{s}$, we found that the mean unbinding time increased to $0.1075 \mathrm{~s}$ from $0.0991 \mathrm{~s}$ with $t_{0}=0 \mathrm{~ms}(\mathrm{P}$ value $<0.0001$, two-tailed t-test). These results highlight the conclusion that the underlying biophysical parameters of the bond are in accessible in histogram analysis without specifically accounting for the loading rate and the detection limit (Supporting Information for more details). 
We further probed the effect of the detection limit by discarding all data from our 50 simulations (same data as used in Table 1) with unbinding time $t<t_{0}=50 \mathrm{~ms}$. We found that 50 to 110 data points of the $N=1000$ were "hidden," on average, by the detection limit $\left(N-n_{0}\right.$, Table 2), representing the shortest 5 to $11 \%$ of the events in the simulated data sets.

The biased nature of the data loss (short events), and the strong effect of loading rate on the extent to which the data is lost (Table 2) makes estimating the number of "hidden" datapoints to from an experimental data set difficult. This data loss significantly affects any quantitative analysis of unbinding time histograms, as described above and detailed in the Supporting Information.

Table 2. Mean fit parameters from simulated slip bond dissociation data sets where short events have been removed

\begin{tabular}{|c|c|c|c|c|c|}
\hline $\begin{array}{c}\text { Loading rate } \\
(\mathrm{pN} / \mathrm{s})\end{array}$ & $\begin{array}{c}n_{0}(\mathrm{mean} \pm \\
\text { s.e.m.) }\end{array}$ & $\begin{array}{c}k_{0, \mathrm{~s}}\left(\mathrm{~s}^{-1}\right)(\mathrm{fit} \pm \\
\text { s.e.m. })\end{array}$ & $\mathrm{P}$ value & $\begin{array}{c}F_{\mathrm{s}}(\mathrm{pN})(\mathrm{fit} \pm \\
\text { s.e.m. })\end{array}$ & $\mathrm{P}$ value \\
\hline $\begin{array}{c}\text { Simulation } \\
\text { parameter }\end{array}$ & 1000 & 1 & - & 1 & - \\
\hline 0 & $950.3 \pm 1.1$ & $1.008 \pm 0.005$ & 0.11 & - & - \\
\hline 0.3 & $951.2 \pm 1.0$ & $0.990 \pm 0.009$ & 0.24 & $1.030 \pm 0.044$ & 0.49 \\
\hline 1 & $950.6 \pm 1.0$ & $0.997 \pm 0.010$ & 0.79 & $1.003 \pm 0.016$ & 0.86 \\
\hline 3 & $946.2 \pm 1.1$ & $1.013 \pm 0.010$ & 0.20 & $1.008 \pm 0.010$ & 0.41 \\
\hline 10 & $937.1 \pm 1.1$ & $0.987 \pm 0.014$ & 0.34 & $0.995 \pm 0.007$ & 0.49 \\
\hline 30 & $890.5 \pm 1.3$ & $0.984 \pm 0.022$ & 0.48 & $0.997 \pm 0.008$ & 0.74 \\
\hline
\end{tabular}

However, we found that the situation was much improved when we fit these data to $n(t)=$ $n_{0} e^{-k_{0, s}\left(t-t_{0}\right)}$ and equation (9) using the number of "detected" unbinding events in each

297 simulation, $n_{0}, f$, and $t_{0}=50 \mathrm{~ms}$ as fixed parameters. We calculated the mean of the 50 sets of fitting parameters for each loading rate (Table 2). We found that the means were not 
significantly different from the parameters used in the simulations, i.e., $k_{0, \mathrm{~s}}=1 \mathrm{~s}^{-1}$ and $F_{\mathrm{s}}=1$ $\mathrm{pN}$ ( $\mathrm{P}$ values $>0.05$ in all cases, Table 2, two-tailed t-tests), and that none of the mean fitting parameters were significantly different from the fitting parameters found using all the data $(\mathrm{P}$

302 values $>0.05$ in all cases, comparing data in Table 1 and Table 2, two-tailed t-tests). Thus, 303 despite the "hidden" data, we showed that our cumulative distribution function-like analysis unambiguously recovers the physical parameters of the slip bonds.

\section{Catch-slip bonds}

306 We also simulated $N=1000$ catch-slip bonds with an unloaded unbinding rate of $k_{0, c}=$ $3071 \mathrm{~s}^{-1}$, catch bond force sensitivity $F_{\mathrm{c}}=1 \mathrm{pN}$, slip bond unloaded unbinding rate $k_{0, \mathrm{~s}}=$ $3080.05 \mathrm{~s}^{-1}$, and slip bond force sensitivity $F_{\mathrm{s}}=1 \mathrm{pN}$ (the same parameters used in Figure $2 \mathrm{f}$, 309 these equate to $\left.F_{\mathrm{c}-\mathrm{s}}=1.5 \mathrm{pN}\right)$ subjected to linearly increasing loads $\left(k_{\mathrm{off}, \mathrm{c}-\mathrm{s}}(F=f t)\right.$ per 310 equation (16) and $n(t)$ per equation (18), Figure 4a), as well as with no loading

$311\left(k_{\mathrm{off}, \mathrm{c}-\mathrm{s}}(F=0)=k_{0, \mathrm{c}}\right.$ per equation (16) and $n(t)=\mathrm{N} e^{-k_{0, \mathrm{c}} t}$, equivalent to the unloaded slip

312 bond case, Figure 3a, dark purple). We found that the nature of the time to detach distribution 313 histograms was a strong function of loading rate. Low external loading rates $(f=0.3 \mathrm{pN} / \mathrm{s}$,

314 Figure $4 \mathrm{~b}$, upper left) exhibited a nearly exponential distribution of unbinding times, like the 315 other bond types we have discussed, and high external loading rates $(f=30 \mathrm{pN} / \mathrm{s}$, Figure $4 \mathrm{~b}$, 316 lower right) corresponded to a large Gaussian-like distribution of unbinding events at longer 317 times. It is in the intermediate loading rate regime that two separate peaks, coming from the 318 separate, significant contributions from the catch bond (short events) and slip bond (long 319 events) behaviors, respectively, emerge (Figure 4b, upper right and lower left).

320 We also plotted the force-free and force-dependent dissociation of simulated slip bonds 321 (same data as Figure 4b) as a function of their time to dissociate $(n(t))$. We fit these data to 322 $n(t)=\mathrm{N} e^{-k_{0, s} t}$ and equation (18a) (Figure 4c, Table S3, and Supporting Information for 
323 details), respectively. As we did with slip bonds, we input the values for $N$ and $f$ as fixed

324 parameters in the fit and repeated the simulations 50 times. Unlike the case of slip bonds, we

325 found that non-linear least squares regression was unable to uniquely determine fitting 326 parameters in multiple of the simulated data sets at lower and higher loading rates (Supporting

327 Information). Of the 50 simulations, we found that only 22 of the simulated data sets were well-

328 fit by equation (18a) for low loading rate $(0.3 \mathrm{pN} / \mathrm{s})$ and 40 of the simulated data sets were well-fit by equation (18a) for high loading rate $(30 \mathrm{pN} / \mathrm{s})$.

330 We suspected that the errors in the fits were due to an "overfitting" of the data. To investigate 331 this hypothesis, we determined whether the force at unbinding was above for below the catch332 slip transition force, $F_{\mathrm{c}-\mathrm{s}}$, for each unbinding event to classify whether the bond dissociated in 333 the catch $\left(F<F_{\mathrm{c}-\mathrm{s}}\right)$ or slip $\left(F>F_{\mathrm{c}-\mathrm{s}}\right)$ regime. We plotted the mean number of bonds 334 dissociating in the slip regime for the 50 simulations with $N=1000$ total bonds (Figure 4d, 335 purple dashed line). We found that most of the bonds dissociated in the catch bond regime 336 (Figure 4d, purple dashed line) when the loading rate was low, corresponding to an exponential-like distribution of unbinding events (Figure 4b, upper left). At high loading rates, the fraction of bonds that dissociated in the slip force regime was high (Figure $4 \mathrm{~d}$, purple dashed line), corresponding to a large Gaussian-like distribution at relatively longer times

340 (Figure 4b, lower right) and similar to slip bonds (Figure 3b, lower right). Together, these data 341 suggest that the slip bond parameters have little effect on the unbinding behaviors at low 342 loading rates and that the catch bond parameter have little effect on the unbind behaviors at 343 high loading rates. Thus, attempting to fit equation (18a), which contains both the slip and 344 catch bond-related parameters, is less effective at low or high loading rates.

345 Moreover, even when the low and high loading rate data are well-fit by equation (18a), i.e., 346 the non-linear least squares regression was able to uniquely determine fitting parameters, we 347 found that the standard errors of those fits tend to be significantly larger than for intermediate 
348 loading rates (Tables 3 and S3, and Supporting Information for more details). Therefore, these

349 results suggest that one must use intermediate loading rates, i.e., loading rates for which a

350 significant number of bonds dissociate in the catch and slip regimes (Figure $4 \mathrm{~d}$, purple dashed

351 line), to determine the biophysical properties of catch-slip bonds.

352 We also calculated the mean detachment time for each force-ramp loading rate condition

353 (Figure 4d, orange solid line). We found that the mean detachment time increased for faster

354 loading rates while the dissociations were dominated by the catch-bond behavior (Figure $4 \mathrm{~d}$,

355 purple dashed line). However, this trend reversed at an intermediate loading rate and the mean

356 detachment time decreased with increasing loading rate (Figure $4 \mathrm{~d}$, orange solid line) as more

357 the dissociations were dominated by the slip-bond behavior (Figure $4 \mathrm{~d}$, purple dashed line).

358 We had found that determining the underlying biophysical parameters of slip bonds from 359 characterizations of the mean unbinding time and associated histograms requires collecting

360 large data sets at multiple loading rates (Supporting Information for more details). However,

361 we found that, to the best of our knowledge, an equivalent analysis did not yield analytical

362 solutions, even with special functions, from which we could calculate the underlying

363 biophysical parameters of catch-slip bonds using the mean unbinding time and associated 364 histograms.

365 Together, this analysis and these data highlight the importance of performing single-

366 molecule experiments at optimized experimental conditions, i.e., ones that enable

367 characterization of all the physics associated with catch-slip bonds, and the utility of our 368 cumulative distribution-like analysis. 

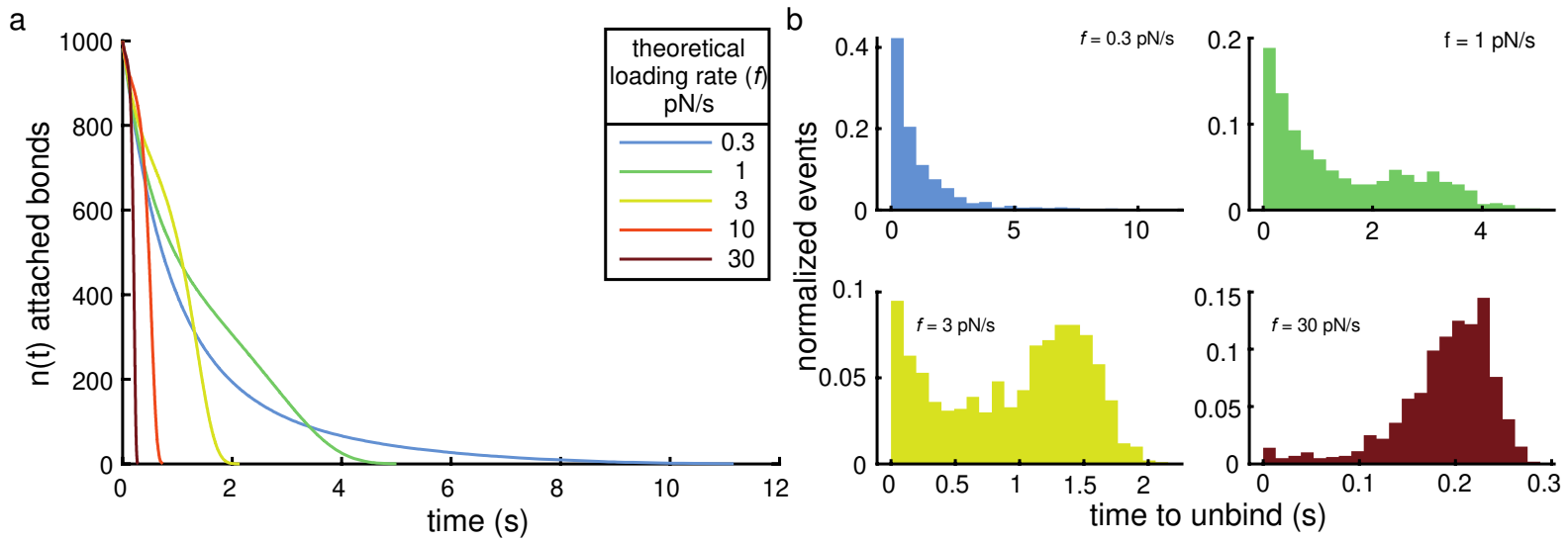

C
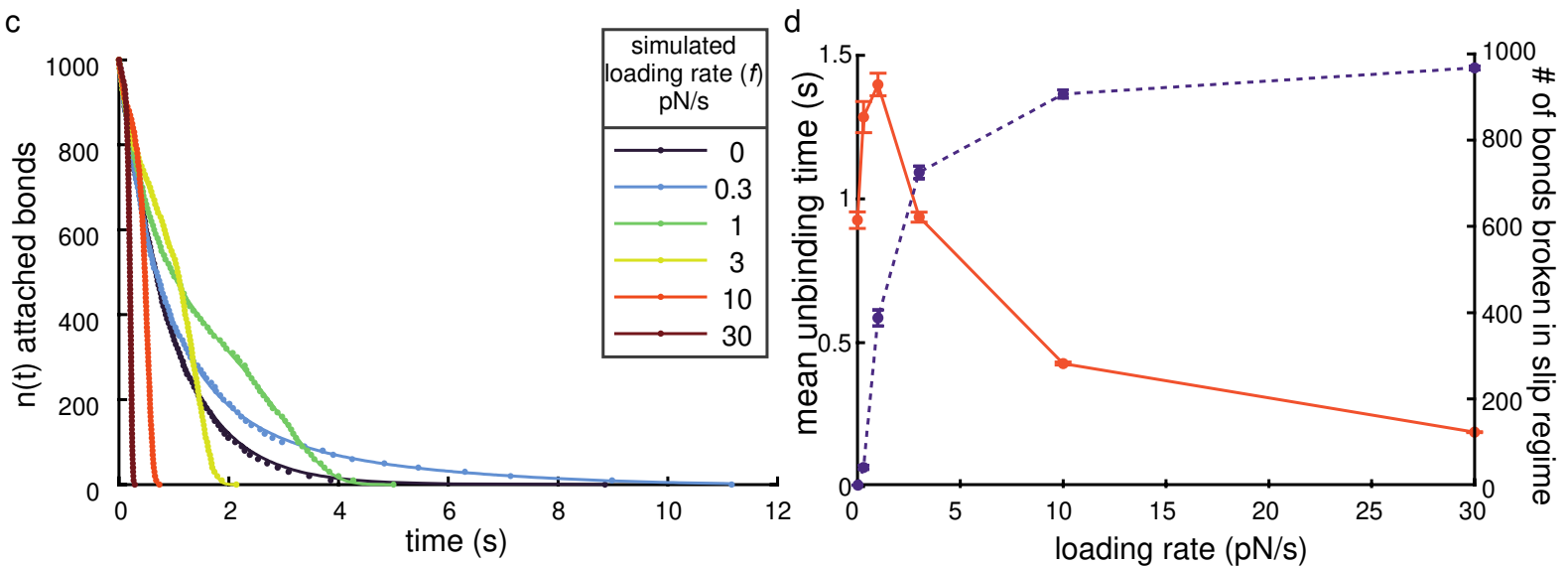

Figure 4. Calculating the force-dependent unbinding rate for catch-slip bonds.

372 As discussed above, noise in force-spectroscopy data can hide dissociations that occur at

373 short times. To investigate the role of experimental noise on the analysis of catch-slip bonds,

374 we excluded simulated dissociation events from our 50 simulations (same data as used in Table

375 3) with unbinding time $t<t_{0}=50 \mathrm{~ms}$. We fit $n(t)$ with equation (18b) to calculate the corresponding bond parameters (Table 4). We found that non-linear least squares regression uniquely determined fitting parameters in fewer of the simulated data sets, particularly at higher

378 loading rates, than when not accounting for experimental noise (Supporting Information). Of

379 the 50 simulations, we found that only 24 of the $f=0.3 \mathrm{pN} / \mathrm{s}, 33$ of the $f=10 \mathrm{pN} / \mathrm{s}$, and 5 of

380 the $f=30 \mathrm{pN} / \mathrm{s}$ simulated data sets were well-fit by equation (18b) (Table 4).

Table 3. Mean fit parameters from simulated catch-slip bond dissociation data sets 


\begin{tabular}{|c|c|c|c|c|c|c|c|c|c|}
\hline $\begin{array}{l}\text { Loading } \\
\text { rate }(\mathrm{pN} / \mathrm{s})\end{array}$ & $\begin{array}{l}\text { Significant } \\
\text { fits }\end{array}$ & $\begin{array}{c}k_{0, \mathrm{c}}\left(\mathrm{s}^{-1}\right) \\
(\mathrm{mean} \pm \\
\text { s.e.m. })\end{array}$ & $\begin{array}{c}\mathrm{P} \\
\text { value }\end{array}$ & $\begin{array}{c}F_{\mathrm{c}}(\mathrm{pN}) \\
(\text { mean } \pm \\
\text { s.e.m. })\end{array}$ & $\begin{array}{c}\mathrm{P} \\
\text { value }\end{array}$ & $\begin{array}{c}k_{0, \mathrm{~s}}\left(\mathrm{~s}^{-1}\right) \\
(\mathrm{mean} \pm \\
\text { s.e.m.) }\end{array}$ & $\begin{array}{c}\mathrm{P} \\
\text { value }\end{array}$ & $\begin{array}{c}F_{\mathrm{s}}(\mathrm{pN}) \\
(\text { mean } \pm \\
\text { s.e.m. })\end{array}$ & $\begin{array}{c}\mathrm{P} \\
\text { value }\end{array}$ \\
\hline $\begin{array}{l}\text { Simulation } \\
\text { parameter }\end{array}$ & 50 & 1 & - & 1 & - & 0.05 & & 1 & - \\
\hline 0 & 50 & $\begin{array}{c}1.0478^{*} \pm \\
0.0054\end{array}$ & 0.69 & - & - & - & - & - & - \\
\hline 0.3 & 22 & $\begin{array}{c}1.0058 \pm \\
0.0171\end{array}$ & 0.74 & $\begin{array}{c}1.1698 \pm \\
0.0698\end{array}$ & 0.02 & $\begin{array}{c}0.0360 \pm \\
0.0160\end{array}$ & 0.39 & $\begin{array}{c}0.6043 \pm \\
0.1441\end{array}$ & 0.01 \\
\hline 1 & 50 & $\begin{array}{c}1.0115 \pm \\
0.0109\end{array}$ & 0.30 & $\begin{array}{c}0.9826 \pm \\
0.0342\end{array}$ & 0.61 & $\begin{array}{c}0.0589 \pm \\
0.0048\end{array}$ & 0.07 & $\begin{array}{c}1.0341 \pm \\
0.0304\end{array}$ & 0.27 \\
\hline 3 & 50 & $\begin{array}{c}1.0146 \pm \\
0.0145\end{array}$ & 0.32 & $\begin{array}{c}1.0467 \pm \\
0.0295\end{array}$ & 0.12 & $\begin{array}{c}0.0485 \pm \\
0.0017\end{array}$ & 0.38 & $\begin{array}{c}0.9878 \pm \\
0.0093\end{array}$ & 0.20 \\
\hline 10 & 47 & $\begin{array}{c}1.0965 \pm \\
0.0326\end{array}$ & 0.005 & $\begin{array}{c}1.0120 \pm \\
0.0547\end{array}$ & 0.82 & $\begin{array}{c}0.0496 \pm \\
0.0016\end{array}$ & 0.98 & $\begin{array}{c}0.9952 \pm \\
0.0065\end{array}$ & 0.46 \\
\hline 30 & 40 & $\begin{array}{c}1.3684 \pm \\
0.0795\end{array}$ & $\begin{array}{c}< \\
0.0001\end{array}$ & $\begin{array}{c}0.8083 \pm \\
0.0924\end{array}$ & .04 & $\begin{array}{c}0.0502 \pm \\
0.0021\end{array}$ & 0.92 & $\begin{array}{c}0.9974 \pm \\
0.0070\end{array}$ & 0.71 \\
\hline
\end{tabular}

$382 * k_{\mathrm{off}, \mathrm{c}-\mathrm{s}}(F=0)=k_{0, \mathrm{c}}+k_{0, \mathrm{~s}}=1.05$ per equation (17) for the parameters used in the

383 simulation

384 Table 4. Mean fit parameters from simulated catch-slip bond dissociation data sets where

385 short events have been removed

\begin{tabular}{|c|c|c|c|c|c|c|c|c|c|c|}
\hline $\begin{array}{l}\text { Loading } \\
\text { rate }(\mathrm{pN} / \mathrm{s})\end{array}$ & $\begin{array}{l}\text { Significant } \\
\text { fits }\end{array}$ & $\begin{array}{c}n_{0} \\
\text { (mean } \pm \\
\text { s.e.m.) }\end{array}$ & $\begin{array}{c}k_{0, \mathrm{c}}\left(\mathrm{s}^{-1}\right) \\
(\text { mean } \pm \\
\text { s.e.m. })\end{array}$ & $\begin{array}{c}\mathrm{P} \\
\text { value }\end{array}$ & $\begin{array}{c}F_{\mathrm{c}}(\mathrm{pN}) \\
(\text { mean } \pm \\
\text { s.e.m. })\end{array}$ & $\begin{array}{c}\mathrm{P} \\
\text { value }\end{array}$ & $\begin{array}{c}k_{0, \mathrm{~s}}\left(\mathrm{~s}^{-1}\right) \\
(\text { mean } \pm \\
\text { s.e.m. })\end{array}$ & $\begin{array}{c}\mathrm{P} \\
\text { value }\end{array}$ & $\begin{array}{c}F_{\mathrm{s}}(\mathrm{pN}) \\
(\text { mean } \pm \\
\text { s.e.m. })\end{array}$ & $\begin{array}{c}\mathrm{P} \\
\text { value }\end{array}$ \\
\hline $\begin{array}{l}\text { Simulation } \\
\text { parameter }\end{array}$ & 50 & & 1 & - & 1 & - & 0.05 & - & 1 & - \\
\hline 0 & 50 & $\begin{array}{c}949.4 \pm \\
1.1\end{array}$ & $\begin{array}{c}1.0484^{*} \pm \\
0.0053\end{array}$ & 0.76 & - & - & - & - & - & - \\
\hline 0.3 & 24 & $\begin{array}{c}948.4 \pm \\
0.8\end{array}$ & $\begin{array}{c}0.9956 \pm \\
0.0164\end{array}$ & 0.79 & $\begin{array}{c}1.1764 \pm \\
0.0678\end{array}$ & 0.02 & $\begin{array}{c}0.0391 \pm \\
0.0150\end{array}$ & 0.47 & $\begin{array}{c}0.6217 \pm \\
0.1397\end{array}$ & 0.01 \\
\hline 1 & 50 & $\begin{array}{c}949.3 \pm \\
1.0\end{array}$ & $\begin{array}{c}1.0149 \pm \\
0.0128\end{array}$ & 0.25 & $\begin{array}{c}0.9944 \pm \\
0.0416\end{array}$ & 0.89 & $\begin{array}{c}0.0594 \pm \\
0.0050\end{array}$ & 0.06 & $\begin{array}{c}1.0348 \pm \\
0.0317\end{array}$ & 0.28 \\
\hline 3 & 50 & $\begin{array}{c}950.6 \pm \\
0.7\end{array}$ & $\begin{array}{c}0.9972 \pm \\
0.0214\end{array}$ & 0.90 & $\begin{array}{c}1.1124 \pm \\
0.0449\end{array}$ & 0.02 & $\begin{array}{c}0.0472 \pm \\
0.0018\end{array}$ & 0.13 & $\begin{array}{c}0.9812 \pm \\
0.0095\end{array}$ & 0.05 \\
\hline
\end{tabular}

Page 21 of 32 


\begin{tabular}{|c|c|c|c|c|c|c|c|c|c|c|}
\hline 10 & 33 & $957.0 \pm$ & $1.3098 \pm$ & 0.01 & $1.1293 \pm$ & 0.27 & $0.0482 \pm$ & 0.30 & $0.9897 \pm$ \\
0.9 & 0.1197 & & 0.1145 & & 0.0017 & & 0.0075 \\
& & 0.9 & & & & & & \\
& & $567.7 \pm$ & $0.9693 \pm$ & 0.93 & $0.7971 \pm$ & 0.41 & $0.0541 \pm$ & 0.45 & $1.0131 \pm$ & 0.46 \\
& & 0.6 & 0.3377 & & 0.2214 & & 0.0049 & & 0.0159 & \\
\hline
\end{tabular}

$386 * k_{\mathrm{off}, \mathrm{c}-\mathrm{s}}(F=0)=k_{0, \mathrm{c}}+k_{0, \mathrm{~s}}=1.05$ per equation (17) for the parameters used in the

387 simulation

388 As we observed for the simulated data without accounting for the effect of experimental

389 noise (Table 3), the cumulative distribution function-like analysis technique was able to resolve

390 the catch-bond fitting parameters reasonably well (despite a $17 \%$, P value $=0.02$ two-tailed t-

391 test, error in resolving the force sensitivity of the catch bond) with low s.e.m. of the fits in low

392 loading rate simulations, and the slip-bond fitting parameters (despite a low number of well-fit

393 data sets) in the high loading rate simulation (Table 4). Additionally, as we observed for the

394 simulated data without accounting for experimental noise (Table 3), catch-bond fitting

395 parameters were not well resolved at high loading rate simulations, and slip-bond fitting

396 parameters were not well resolved at low loading rates (Table 4). Like we found with slip

397 bonds, none of the fit parameters differed significantly from those found when accounting for

398 the complete simulated data set ( $\mathrm{p}$ values $>0.05$, two-sample two-tailed t-tests, Tables 3 and

399 4), again highlighting the benefit of our method when analyzing noisy experimental data.

400 These data again serve to highlight the effectiveness of the cumulative distribution-like

401 analysis to resolve the biophysical parameters associated with catch-slip bonds, even when

402 experimental noise hides short events. However, the reduction in the number of well-fit data

403 sets, and the increased sensitivity of the catch bond parameters to the loading rate when short

404 events are hidden by noise, does strongly suggest that loading rates must be chosen carefully,

405 particularly for catch-slip bonds with relatively low catch-slip transition force, $F_{\mathrm{c}-\mathrm{s}}$. 
407 We presented an analytically derived cumulative distribution-like function of unbinding 408 events $(n(t))$ for various biological macromolecular bond types when subject to force. We

409 showed how an $n(t)$, cumulative distribution function-like, based approach can be used to 410 analyze force-dependent dissociation force spectroscopy data. We demonstrated the benefits 411 and limitations of the technique using stochastic simulations (Gillespie algorithm) of slip and 412 catch-slip bonds. The approach can determine the detachment rate and force sensitivity of 413 biological macromolecular bonds from force spectroscopy experiments by explicitly 414 accounting for loading rate more efficiently than histogram-based analyses. This analysis 415 approach requires fewer, smaller data sets than alternative approaches. Additionally, the 416 approach returns similar (not statistically different) results when short events are hidden by 417 noisy data. We suggest that this approach provides an improved systematic and quantitative 418 method to distinguishing various bond types and characterizing their underlying biophysical 419 properties.

420 We also analyzed the effect of using a range of loading rates to probe the force-dependent unbinding of biological macromolecules. Our simulated data suggests that this analysis of slipbonds is largely insensitive to loading rate. However, care must be taken to ensure a significant

423 fraction of the bonds dissociate in both the catch- and slip-bond force regimes, when the bond 424 is a catch-slip bond. Thus, if an experiment is being done on a bond of unknown type, multiple 425 loading rates are necessary to ensure all possible molecular dissociation pathways are 426 sufficiently sampled in the experiments to resolve their underlying biophysical parameters.

427 In summary, our approach provides a framework for an improved analysis of force428 dependent biological macromolecular dissociation force spectroscopy data. It explicitly accounts for the loading rate, which may be complicated by optical tweezer trap stiffness and 
430 biological macromolecular stiffness, to distinguish between and fully characterize the 431 biophysical properties of protein-protein and protein-ligand bonds.

\section{References}

433 1. Thomas, W. E., Vogel, V. \& Sokurenko, E. Biophysics of Catch Bonds. Annual Review of $434 \quad$ Biophysics 37, 399-416 (2008).

2. Howard, J. Mechanics of Motor Proteins and the Cytoskeleton. (Sinauer Associates, $436 \quad$ Publishers, 2001).

3. Marshall, B. T. et al. Direct observation of catch bonds involving cell-adhesion molecules. Nature 423, 190-193 (2003).

4. Priest, A. V., Shafraz, O. \& Sivasankar, S. Biophysical basis of cadherin mediated cell-cell adhesion. Experimental Cell Research 358, 10-13 (2017).

5. Rakshit, S. \& Sivasankar, S. Biomechanics of cell adhesion: how force regulates the lifetime of adhesive bonds at the single molecule level. Physical Chemistry Chemical Physics 16, 2211-2223 (2014).

6. Hong, J. et al. A TCR mechanotransduction signaling loop induces negative selection in the thymus. Nature Immunology 19, 1379-1390 (2018).

7. Hoffman, B. D., Grashoff, C. \& Schwartz, M. A. Dynamic molecular processes mediate cellular mechanotransduction. Nature 475, 316-323 (2011).

8. Hoekstra, T. P. et al. Switching between Exonucleolysis and Replication by T7 DNA Polymerase Ensures High Fidelity. Biophysical Journal 112, 575-583 (2017).

9. Biebricher, A. S. et al. The impact of DNA intercalators on DNA and DNA-processing enzymes elucidated through force-dependent binding kinetics. Nature Communications 6 , $7304(2015)$. 
10. Dembo, M., Torney, D. C., Saxman, K. \& Hammer, D. The Reaction-Limited Kinetics of Membrane-to-Surface Adhesion and Detachment. Proceedings of the Royal Society of London. Series B, Biological Sciences 234, 55-83 (1988).

11. Sarangapani, K. K. et al. Low Force Decelerates L-selectin Dissociation from P-selectin Glycoprotein Ligand-1 and Endoglycan *. Journal of Biological Chemistry 279, 22912298 (2004).

12. Scheuermann, J., Viti, F. \& Neri, D. Unexpected observation of concentration-dependent dissociation rates for antibody-antigen complexes and other macromolecular complexes in competition experiments. Journal of Immunological Methods 276, 129-134 (2003).

13. Hinterdorfer, P., Schilcher, K., Baumgartner, W., Gruber, H. J. \& Schindler, H. A mechanistic study of the dissociation of individual antibody-antigen pairs by atomic force microscopy. Nanobiology 4, 177 (1998).

14. Kulin, S., Kishore, R., Hubbard, J. B. \& Helmerson, K. Real-Time Measurement of Spontaneous Antigen-Antibody Dissociation. Biophysical Journal 83, 1965-1973 (2002).

15. Dembo, M. On peeling an adherent cell from a surface. Lect. Math. Life Sci. 24, (1994).

16. Bell, G. I. Models for the specific adhesion of cells to cells. Science 200, 618-627 (1978).

17. Dahlke, K., Zhao, J., Sing, C. E. \& Banigan, E. J. Force-Dependent Facilitated Dissociation Can Generate Protein-DNA Catch Bonds. Biophys J 117, 1085-1100 (2019).

18. Nishizaka, T., Miyata, H., Yoshikawa, H., Ishiwata, S. \& Kinosita, K. Unbinding force of a single motor molecule of muscle measured using optical tweezers. Nature 377, 251-254 (1995).

19. Jannasch, A., Bormuth, V., Storch, M., Howard, J. \& Schäffer, E. Kinesin-8 Is a Low476 Force Motor Protein with a Weakly Bound Slip State. Biophysical Journal 104, 2456-2464 (2013). 
20. Bormuth, V., Varga, V., Howard, J. \& Schäffer, E. Protein Friction Limits Diffusive and Directed Movements of Kinesin Motors on Microtubules. Science 325, 870-873 (2009).

21. Capitanio, M. \& Pavone, F. S. Interrogating Biology with Force: Single Molecule HighResolution Measurements with Optical Tweezers. Biophysical Journal 105, 1293-1303 (2013).

22. Honarmandi, P., Lee, H., J. Lang, M. \& D. Kamm, R. A microfluidic system with optical laser tweezers to study mechanotransduction and focal adhesion recruitment. Lab on a Chip 11, 684-694 (2011).

23. Grashoff, C. et al. Measuring mechanical tension across vinculin reveals regulation of focal adhesion dynamics. Nature 466, 263-266 (2010).

24. Bustamante, C., Alexander, L., Maciuba, K. \& Kaiser, C. M. Single-Molecule Studies of Protein Folding with Optical Tweezers. Annual Review of Biochemistry 89, 443-470 (2020).

25. Smith, S. B., Cui, Y. \& Bustamante, C. Overstretching B-DNA: The Elastic Response of Individual Double-Stranded and Single-Stranded DNA Molecules. Science 271, 795-799 (1996).

26. Wang, M. D. et al. Force and Velocity Measured for Single Molecules of RNA Polymerase. Science 282, 902-907 (1998).

27. Rao, L., Berger, F., Nicholas, M. P. \& Gennerich, A. Molecular mechanism of cytoplasmic dynein tension sensing. Nature Communications 10, 3332 (2019).

28. Nicholas, M. P. et al. Cytoplasmic dynein regulates its attachment to microtubules via nucleotide state-switched mechanosensing at multiple AAA domains. PNAS 112, 6371$6376(2015)$

29. Andreasson, J. O. et al. Examining kinesin processivity within a general gating framework. eLife 4, e07403 (2015). 
30. Evans, E. Probing the Relation Between Force-Lifetime-and Chemistry in Single Molecular Bonds. Annual Review of Biophysics and Biomolecular Structure 30, 105-128 (2001).

31. Berger, F., Klumpp, S. \& Lipowsky, R. Force-Dependent Unbinding Rate of Molecular Motors from Stationary Optical Trap Data. Nano Lett. 19, 2598-2602 (2019).

32. Trefethen, L. N. Approximation Theory and Approximation Practice, Extended Edition. (SIAM, 2019).

33. Corless, R. M. \& Fillion, N. Polynomial and Rational Interpolation. in A Graduate Introduction to Numerical Methods: From the Viewpoint of Backward Error Analysis (eds. Corless, R. M. \& Fillion, N.) 331-401 (Springer, 2013). doi:10.1007/978-1-4614-8453$0 \_8$.

34. Mathematica. (Wolfram Research, Inc., 2021).

35. Pereverzev, Y. V., Prezhdo, O. V., Forero, M., Sokurenko, E. V. \& Thomas, W. E. The Two-Pathway Model for the Catch-Slip Transition in Biological Adhesion. Biophysical Journal 89, 1446-1454 (2005).

36. Prezhdo, O. V. \& Pereverzev, Y. V. Theoretical Aspects of the Biological Catch Bond. Acc. Chem. Res. 42, 693-703 (2009).

37. Pereverzev, Y. V., Prezhdo, O. V., Thomas, W. E. \& Sokurenko, E. V. Distinctive features of the biological catch bond in the jump-ramp force regime predicted by the two-pathway model. Phys. Rev. E 72, 010903 (2005).

38. Rakshit, S., Zhang, Y., Manibog, K., Shafraz, O. \& Sivasankar, S. Ideal, catch, and slip bonds in cadherin adhesion. PNAS 109, 18815-18820 (2012).

39. MATLAB. (The MathWorks, Inc., 2021).

40. Gillespie, D. T. A general method for numerically simulating the stochastic time evolution of coupled chemical reactions. Journal of Computational Physics 22, 403-434 (1976). 
527 41. Phillips, R., Kondev, J. \& Theriot, J. Physical Biology of the Cell. (Garland Science, 2013).

528 Acknowledgements

529 AP was supported by a Clemson Research Fellows R-Initiative Award from Clemson 530 University. This work was supported by the National Institute of Allergy and Infectious

531 Diseases (NIAID) of the National Institutes of Health under award number R15AI137979, by

532 the National Institute of General Medical Sciences (NIGMS) of the National Institutes of 533 Health under award number P30 GM131959, and by Clemson University. We would like to

534 thank Ashok Pabbathi for his sample data (Figure 1b) and Ashok Pabbathi and Subash Godar 535 for their insightful discussions. Additionally, we thank Marija Zanic for reading and 536 commenting on early drafts of the manuscript.

\section{Author contributions}

538 A.P. and J.A. contributed to the conceptualization of the study, performed the analytical and 539 computational work, analyzed the results, and revised the manuscript. A.P. drafted the first 540 draft of the manuscript. J.A. supervised the project.

\section{Additional Information}

542 We declare that the authors have no competing interests as defined by Nature Research, or

543 other interests that might be perceived to influence the results and/or discussion reported in this 544 paper.

545 Figure legends

546 Figure 1. Microtubule-associated protein dissociation from a microtubule in a force-ramp

547 optical trapping assay. (a) Schematic of a microtubule (red and orange circles) bound to a 
stage that moves (with velocity, $v$, black arrow) and pulls a bead conjugated to a microtubuleassociated protein (MAP, green) out of the trap center (with effective stiffness of the trap and MAP, $\kappa$ ). The force that the trap exerts on the bead (orange arrow) increases at a rate of $f=$ $\kappa v$ (thus $F(t)=f t$ ) until the MAP stochastically unbinds from the microtubule, which causes it to return to the trap center. The force-dependent unbinding rate constant, $k_{\text {off }}(F)$, governs the biophysics of unbinding. (b) A typical force spectroscopy trace (gray = raw data, black= filtered data) from a force-ramp optical tweezer assay showing the force as a function of time during a biological macromolecular binding (initial binding, purple dot) and unbinding (final unbinding, orange dot) event. The unbinding force and bound times (orange arrows) are used to determine $k_{\text {off }}(F)$ for the MAP-microtubule interaction. An event is "detected" when the filtered signal exceeds 5-times the standard deviation of the noise (dashed lines).

Figure 2. Analytical models for the force-dependent unbinding constant of slip-ideal, catch-ideal, and catch-slip bonds. (a) The piecewise function that explicitly models a slipideal bond (equation (10)) normalized by the unbinding constant at zero force and plotted as a function of force normalized by the slip-ideal transition force for the case of $F_{\mathrm{s}-\mathrm{i}}=F_{\mathrm{S}}$. (b) A Michaelis-Menten or Langmuir absorption-like approximation of the slip-ideal bond (equation (S1)) normalized by the ideal bond's unbinding constant and plotted as a function of the force normalized by the Michaelis constant-like characteristic force. (c) A sigmoidal approximation of a slip-ideal bond (equation (S3)) where $k_{\mathrm{a}}=2, k_{\mathrm{b}}=1, F_{\mathrm{a}}=\frac{1}{5}$ and $F_{\mathrm{b}}=\frac{3}{5}$. (d) A rational

567 interpolation approximation (equation (S5), solid line) of the piecewise function from panel (a) 568 (dashed line) for a slip-ideal bond with $F_{\mathrm{s}-\mathrm{i}}=F_{\mathrm{s}}=1$, which is equivalent to $\beta=1$ 569 (Supplementary information), normalized by the slip-bond unbinding constant, and plotted as a function of the force normalized by the characteristic force of the slip ideal transition, i.e., 
$\frac{k_{\mathrm{off}, \mathrm{s}-\mathrm{i}}(F(t))}{k_{0, \mathrm{~s}}}=\frac{1-0.42 \frac{F}{F_{\mathrm{s}-\mathrm{i}}}+0.27\left(\frac{F}{F_{\mathrm{s}-\mathrm{i}}}\right)^{2}}{1-1.35 \frac{F}{F_{\mathrm{s}-\mathrm{i}}}+0.84\left(\frac{F}{F_{\mathrm{s}-\mathrm{i}}}\right)^{2}-0.15\left(\frac{F}{F_{\mathrm{s}-\mathrm{i}}}\right)^{3}}$ per Table S1. (e) The piecewise function (blue,

572 equation (13)) that explicitly models a catch-ideal bond, as well as functional approximations

573 of that function (red, yellow, green, equation (20)), normalized by the characteristic slip-bond 574 unbinding constant and plotted as a function of force normalized by the force of the catch-bond

575 for the representative case of $k_{0, \mathrm{c}}=3 k_{0, \mathrm{i}}$. (f) The piecewise function (blue, equation (16)) and

576 sum formulation (yellow, equation (17)) of a model for the force-dependent unbinding constant

577 of the catch-slip bond. In both cases, we used $k_{0, \mathrm{c}}=1 \mathrm{~s}^{-1}, F_{\mathrm{c}}=1 \mathrm{pN}, k_{0, \mathrm{~s}}=0.05 \mathrm{~s}^{-1}$, and $F_{\mathrm{s}}=$

$5781 \mathrm{pN}$ as an example set of parameters. Given these parameters, $F_{\mathrm{c}-\mathrm{s}}=1.50 \mathrm{pN}$.

Figure 3. Calculating the force-dependent unbinding rate for slip bonds. (a) $n(t)=$

$N e^{-k_{0, s} t}$ plotted for no loading (dark purple line) and equation (5) plotted for various loading rates (colored lines) with $N=1000$ slip bonds that have an unloaded unbinding rate $k_{0, s}=1$ $\mathrm{s}^{-1}$ and force sensitivity $F_{\mathrm{S}}=1 \mathrm{pN}$. (b) Histograms of the time to unbind from a typical, $N=$ 1000, slip bond simulation with parameters as in panel (a) for various loading rates, as indicated. (c) Number of bound slip bonds as a function of time from example simulations at various loading rates, as indicated. Inset shows detail highlighting the characteristic shape of $n(t)$ for higher loading rates $(3 \mathrm{pN} / \mathrm{s}$ yellow, $10 \mathrm{pN} / \mathrm{s}$ orange, and $30 \mathrm{pN} / \mathrm{s}$ dark red $)$. The data $($ dots $)$ were fitted to $n(t)=N e^{-k_{0, s} t}$ for $f=0($ dark purple line $)$ and equation (5) for the others (lines). In both cases, see Table S2 for the fit parameters. The same example simulation data were used as in panel (b), where applicable. (d) Mean unbinding time of 50 simulations, with $N=1000 \mathrm{slip}$ bonds each, as a function of loading rate. The error bars represent s.e.m. Equation (18a) plotted for various loading rates (colored lines) with $N=1000$ slip bonds an unloaded unbinding rate of $k_{0, \mathrm{c}}=1 \mathrm{~s}^{-1}$, catch bond force sensitivity of $F_{\mathrm{c}}=1 \mathrm{pN}$, slip bond 
unbinding rate of $k_{0, \mathrm{~s}}=0.05 \mathrm{~s}^{-1}$, and slip bond force sensitivity of $F_{\mathrm{s}}=1 \mathrm{pN}$, as in Figure $2 \mathrm{f}$.

(b) Histograms of the time to unbind from a typical $N=1000$ slip bond simulation with parameters as in (a) for various loading rates, as indicated. (c) Number of bound catch-slip bonds as a function of time for example simulations at various loading rates, as indicated. The data (dots) were fit to $n(t)=N e^{-k_{0, c} t}$ for $f=0$ (dark purple line) and equation (18a) for the others (lines). The equations were well-fit to the data in all of the example cases shown here, see Table S3 for the fit parameters and Supporting Information for more detail. The same example simulation data were used as in panel (b), where applicable. (d) Mean unbinding time (orange solid line, left axis) and mean number of bonds broken in the slip bond regime (purple dashed line, right axis) of 50 simulations, with $N=1000$ catch-slip bonds each, as a function of loading rate. The error bars represent the s.e.m. on both data sets.

Table 3: Mean fit parameters simulated slip bond dissociation data sets. Reported values represent the mean \pm s.e.m. of the fit parameters for 50 sets of $N=1000$ simulated slip bond

607 dissociations under the increasing load rate, as indicated. In all cases, $\mathrm{P}$ values are calculated 608 for two-tailed t-tests. events have been removed. Reported values represent the mean \pm s.e.m. of the fit parameters

611 for the same 50 sets of simulated slip bond dissociation data as in Table 1, but where short events corresponding to those that are indistinguishable from experimental noise have been removed. In all cases, $\mathrm{P}$ values are calculated for two-tailed t-tests.

Table 3. Mean fit parameters from simulated catch-slip bond dissociation data sets. 
617 significant. The number of significant fits is indicated. In all cases, $\mathrm{P}$ values are calculated for 618 two-tailed t-tests.

619 Table 4. Mean fit parameters from simulated catch-slip bond dissociation data where

620 short events have been removed. Reported values represent the mean \pm s.e.m. of the fit 621 parameters for the same 50 sets of simulated slip bond dissociation data for which the fits were 622 significant, as in Table 2, but where short events corresponding to those that are 623 indistinguishable from experimental noise have been removed. The number of significant fits 624 is indicated. In all cases, P values are calculated for two-tailed t-tests. 


\section{Supplementary Files}

This is a list of supplementary files associated with this preprint. Click to download.

- Pauletal.Supplementarylnformationv12.pdf 